\title{
Solution of Fractional Order Equations in the Domain of the Mellin Transform
}

\author{
Sunday Emmanuel Fadugba* \\ Department of Mathematics, Ekiti State University, Ado Ekiti, Nigeria
}

\begin{abstract}
This paper presents the Mellin transform for the solution of the fractional order equations. The Mellin transform approach occurs in many areas of applied mathematics and technology. The Mellin transform of fractional calculus of different flavours; namely the Riemann-Liouville fractional derivative, Riemann-Liouville fractional integral, Caputo fractional derivative and the Miller-Ross sequential fractional derivative were obtained. Three illustrative examples were considered to discuss the applications of the Mellin transform and its fundamental properties. The results show that the Mellin transform is a good analytical method for the solution of fractional order equations.
\end{abstract}

Keywords: Caputo fractional derivative, Fractional calculus, Mellin transform, Miller-Ross fractional derivative, Riemann-Liouville fractional derivative, Riemann-Liouville fractional integral

Article History :

Received: 22 June 2019

Received in revised form: 24 August 2019

Accepted for publication: 18 September 2019

Published: 30 December 2019

(C)2019 Journal of the Nigerian Society of Physical Sciences. All Rights Reserved. Communicated by: T. Latunde

\section{Introduction}

The use of fractional derivatives and integrals provides a powerful tool for incorporating history due to its non-local nature [1].

Fractional calculus has gained much attention due to the fact that fractional differential equations provide an excellent instrument for the description of many practical dynamics phenomena emanating from mathematical finance, economics, biology, physics, just to mention a few, see Ref. [2].

The numerical solution of differential equations of integer order has for a long time been a standard topic in numerical and computational mathematics. However, in spite of a large number of recently formulated applied problems, the state of the art is far less advanced for fractional order differential equations [3].

For various methods of solving fractional differential equations see; [4-9], just to mention a few. In this paper a survey of the applications of the Mellin transform in the solution of fractional calculus of different flavours is presented. It covers the widely known flavours, such as Riemann-Liouville fractional derivative, Riemann-Liouville fractional integral, Caputo fractional derivative and the Miller-Ross sequential fractional derivative. The rest of the paper is organized as follows. Section Two presents some definitions of terms. Section Three presents the main results for the Mellin transform of fractional operators of different flavours. In Section Four, three illustrative examples were presented. Section Five concludes the paper.

\footnotetext{
${ }^{*}$ Corresponding Author Tel. No: +2348067032044

Email address: sunday .fadugba@eksu.edu.ng (Sunday Emmanuel Fadugba)
} 


\section{Preliminaries}

Definition 1 Let $f(t)$ be locally Lebesgue integrable over $(0, \infty)$. The Mellin transform of $f(t)$ denoted by $F(\omega)$ is defined as

$$
F(\omega)=\mathcal{M}(f(t), \omega)=\int_{0}^{\infty} f(t) t^{\omega-1} d t
$$

where $\omega \in \mathbb{C}, \omega$ is complex, such that $a_{1}<\mathfrak{R}(\omega)<a_{2}$. The Mellin transform (1) exists if the function $f(t)$ is piecewise continuous in every closed interval $[a, b] \subset(0, \infty)$ and

$$
\int_{0}^{1}|f(t)| t^{a_{1}-1} d t<\infty, \quad \int_{1}^{\infty}|f(t)| t^{a_{2}-1} d t<\infty
$$

Definition 2 Let $f(t)$ be integrable with fundamental strip $(x, y)$. If $b$ is such that $x<b<y$ and $F(\omega=b+i t)=\mathcal{M}(f(t) ; \omega)$ is integrable, the inverse Mellin transform is defined as

$$
f(t)=\frac{1}{2 \pi i} \int_{b-i \infty}^{b+i \infty} F(\omega) t^{-\omega} d \omega, t \in(0, \infty)
$$

The integration contour in (3) is called the Bromwich contour. The fundamental properties of the Mellin transform are presented in the following results.

Theorem 1. Let $f(t)$ be Mellin transformable function defined on $\mathbb{R}_{+}$. If differentiation under integral sign is permitted, then

(a) $\mathcal{M}\left(t^{d} f(t) ; \omega\right)=F(\omega+d)$

(b) $\mathcal{M}(f(t) * g(t) ; \omega)=F(\omega) G(1-\omega)$

(c) $\mathcal{M}\left(t^{\lambda} \int_{0}^{\infty} \tau^{\mu} f(\tau t) g(\tau) d \tau ; \omega\right)=F(\omega+\lambda) G(1-\omega-\lambda+\mu)$

(d) $\mathcal{M}\left(f^{n}(t) ; \omega\right)=\frac{\Gamma(1-\omega+n)}{\Gamma(1-\omega)} F(\omega-n)$

(e) $\mathcal{M}\left(t^{n} f^{n}(t) ; \omega\right)=(-\omega)^{n} F(\omega)$

(f) $\left(\frac{d}{d t}\right)^{n} \mathcal{M}(f(t) ; \omega)=\int_{0}^{\infty} f(t)(\log t)^{n} t^{\omega-1}$ $=\mathcal{M}\left(f(t)(\log t)^{n} ; \omega-1\right)$

(g) $\mathcal{M}\left(\int_{0}^{t} f(\tau) d \tau ; \omega\right)=-\frac{1}{\omega} F(\omega+1)$

For more details see Refs. [3, 10, 11], just to mention a few.

Definition 3 A real function $f(t), t>0$ is said to be in space $\mathbb{C}_{\mu}, \mu \in \mathbb{R}$ if there exists a real number $\rho>\mu$, such that $f(t)=t^{\rho} f_{1}(t)$ where $f(t) \in \mathbb{C}(0,1)$, and it is said to be in space $\mathbb{C}_{\mu}^{n}$ if $f^{n} \in \mathbb{C}_{\mu}, n \in N$

Definition 4 The Riemann-Liouville fractional derivative operator of order $\alpha>0$ of a function $f(t)$ is defined as

$$
\begin{aligned}
& { }_{0} D_{t}^{\alpha} f(t)=\frac{1}{\Gamma(n-\alpha)} \int_{0}^{t}(t-\tau)^{n-\alpha-1} f(\tau) d \tau, \\
& \alpha \in(n-1, n)
\end{aligned}
$$

Definition 5 The Riemann-Liouville fractional integral operator of order $\alpha \geq 0$ of a function $f \in \mathbb{C}_{\mu}, \mu \geq 1$ is defined as

$$
\begin{aligned}
J^{\alpha} f(t) & ={ }_{0} D_{t}^{-\alpha} f(t) \\
& =\frac{1}{\Gamma(\alpha)} \int_{0}^{t}(t-\tau)^{\alpha-1} f(\tau) d \tau, \alpha>0, \tau>0
\end{aligned}
$$

The following result gives some of the properties of the RiemannLiouville fractional operator.
Theorem 2. If $\alpha, \beta \in \mathbb{R}_{+}$, then
(a) ${ }_{0} D_{t}^{\alpha}\left[{ }_{0} D_{t}^{-\beta} f(t)\right]={ }_{0} D_{t}^{\alpha-\beta} f(t)$
(b) $J^{\alpha} J^{\beta} f(t)=J^{\alpha+\beta} f(t)$
(c) $J^{\alpha} J^{\beta} f(t)=J^{\beta} J^{\alpha} f(t)$
(d) $J^{0} f(t)=f(t)$

Definition 6 The Caputo fractional derivative of the function $f \in \mathbb{C}_{-1}^{n}, n \in \mathbb{N}$ is defined as

$$
\begin{aligned}
& { }_{0}^{c} D_{t}^{\alpha} f(t)=\frac{1}{\Gamma(n-\alpha)} \int_{0}^{t}(t-\tau)^{n-\alpha-1} f^{n}(\tau) d \tau, \\
& \alpha \in(n-1, n]
\end{aligned}
$$

The properties of the Caputo fractional derivative were summarized in the following result.

Theorem 3. If $\alpha \in(n-1, n], n \in \mathbb{N}, f \in \mathbb{C}_{-1}^{n}, \mu>-1$, then

$$
\begin{aligned}
& \text { (a) } J^{\alpha}\left[{ }_{0}^{c} D_{t}^{\alpha} f(t)\right]=f(t)-\sum_{j=0}^{n-1} f^{j}(0)\left(\frac{t^{j}}{j !}\right) \\
& \text { (b) }{ }_{0}^{c} D_{t}^{\alpha}\left[J^{\alpha} f(t)\right]=f(t)
\end{aligned}
$$

Definition 7 The Miller-Ross sequential fractional derivative is defined as

$$
\begin{aligned}
& a D_{t}^{\sigma_{n}} \equiv a D_{t}^{\alpha_{n}} a D_{t}^{\alpha_{n}-1} \ldots a D_{t}^{\alpha_{1}} \\
& a D_{t}^{\sigma_{n}-1} \equiv a D_{t}^{\alpha_{n}-1} \ldots a D_{t}^{\alpha_{1}}
\end{aligned}
$$

where

$$
\sigma_{n}=\sum_{j=1}^{n} \alpha_{j}, \quad \alpha_{j} \in(0,1], \quad(j=1,2, \ldots, n)
$$

\section{Mellin transform of fractional operators of different flavours}

This section presents the main results generated for the Mellin transform of fractional operators of different flavours.

\subsection{Mellin transform of the Riemann-Liouville fractional deriva- tive and integral operators}

The Mellin transform of the Riemann-Liouville fractional derivative and integral operators are given in the following results.

Theorem 4. Let $f(t)$ be Mellin transformable function, where $0 \leq n-1<\alpha<n, t>0$, then the Mellin transform of the Riemann-Liouville fractional derivative is obtained as

$$
\mathcal{M}\left({ }_{0} D_{t}^{\alpha} f(t) ; \omega\right)=\frac{\Gamma(1-\omega+\alpha)}{\Gamma(1-\omega)} F(\omega-\alpha)
$$

Proof: Take $0 \leq n-1<\alpha<n, t>0$, where $\alpha$ is the order. From the definition of the Riemann-Liouville fractional derivative in (4), one can write that

$$
\begin{aligned}
{ }_{0} D_{t}^{\alpha} f(t) & =\frac{1}{\Gamma(n-\alpha)} \int_{0}^{t}(t-\tau)^{n-\alpha-1} f(\tau) d \tau \\
& =\frac{d^{n}}{d t^{n}}{ }_{0} D_{t}^{-(n-\alpha)} f(t)
\end{aligned}
$$


Taking the Mellin transform of (11) yields

$$
\begin{aligned}
\mathcal{M}\left({ }_{0} D_{t}^{\alpha} f(t) ; \omega\right) & =\int_{0}^{\infty} \frac{d^{n}}{d t^{n}} h(t) t^{\omega-1} d t \\
& =\int_{0}^{\infty} h^{n}(t) t^{\omega-1} d t
\end{aligned}
$$

with

$$
h(t)={ }_{0} D_{t}^{-(n-\alpha)} f(t)
$$

By means of the derivative property of the Mellin transform and simplifying further, (12) becomes

$$
\begin{aligned}
\mathcal{M}\left({ }_{0} D_{t}^{\alpha} f(t) ; \omega\right)= & \sum_{j=0}^{n-1} \frac{\Gamma(1-\omega+j)}{\Gamma(1-\omega)}\left[{ }_{0} D_{t}^{\alpha-j-1} f(t) t^{\omega-j-1}\right]_{0}^{\infty} \\
& +\frac{\Gamma(1-\omega+\alpha)}{\Gamma(1-\omega)} F(\omega-\alpha)
\end{aligned}
$$

Equation (14) can be written in a simplified form as

$$
\begin{aligned}
\mathcal{M}\left({ }_{0} D_{t}^{\alpha} f(t) ; \omega\right)= & {\left[{ }_{0} D_{t}^{\alpha-1} f(t) t^{\omega-1}\right]_{0}^{\infty} } \\
& +\frac{\Gamma(1-\omega+j)}{\Gamma(1-\omega)} F(\omega-\alpha)
\end{aligned}
$$

for $\alpha \in(0,1)$. Since the first term in the RHS of (15) becomes zero, (10) is established. This completes the proof.

Theorem 5. Let $f(t)$ be Mellin transformable function, where $0 \leq n-1<\alpha<n, t>0$, then the Mellin transform of the Riemann-Liouville fractional integral is obtained as

$$
\begin{aligned}
& \mathcal{M}\left(J^{\alpha} f(t) ; \omega\right)=\mathcal{M}\left({ }_{0} D_{t}^{-\alpha} f(t) ; \omega\right)= \\
& \frac{\Gamma(1-\omega-\alpha)}{\Gamma(1-\omega)} F(\omega+\alpha)
\end{aligned}
$$

Proof: By means of variable transformation $\tau=t \rho, d \tau=t d \rho$, the Riemann-Liouville fractional integral operator given by (5) as

$$
J^{\alpha} f(t)=\frac{1}{\Gamma(\alpha)} \int_{0}^{t}(t-\tau)^{\alpha-1} f(\tau) d \tau, \alpha>0, \tau>0
$$

yields

$$
J^{\alpha} f(t)=\frac{t^{\rho}}{\Gamma(\alpha)} \int_{0}^{\infty} f(t \rho) g(\rho) d \rho
$$

where

$$
g(t)= \begin{cases}(1-t)^{\alpha-1}, & 0 \leq t<1 \\ 0, & t \geq 1\end{cases}
$$

The Mellin transform of (18) is the Euler Beta function of the form

$$
\mathcal{M}(g(t) ; \omega)=\mathcal{B}(\alpha, \omega)=\frac{\Gamma(\alpha) \Gamma(\omega)}{\Gamma(\alpha+\omega)}
$$

By means of (17) and (19), the Mellin transform of the RiemannLiouville fractional integral operator is obtained as

$$
\mathcal{M}\left(J^{\alpha} f(t) ; \omega\right)=\frac{1}{\Gamma(\alpha)} F(\omega+\alpha) \mathcal{B}(\alpha, 1-\omega-\alpha)
$$

with

$$
\mathcal{B}(\alpha, 1-\omega-\alpha)=\frac{\Gamma(\alpha) \Gamma(1-\omega-\alpha)}{\Gamma(1-\omega)}
$$

\subsection{Mellin transform of the Caputo fractional derivatives}

The following result gives the Mellin transform of the Caputo fractional derivatives.

Theorem 6. Let $f(t)$ be Mellin transformable function, where $0 \leq n-1<\alpha \leq n, t>0$ and $\alpha$ is the order, then the Mellin transform of the Caputo fractional derivatives is given by

$$
\mathcal{M}\left({ }_{0}^{c} D_{t}^{\alpha} f(t) ; \omega\right)=\frac{\Gamma(1-\omega+\alpha)}{\Gamma(1-\omega)} F(\omega-\alpha)
$$

Proof: The Caputo fractional derivative given by (6) can be written as

$$
\begin{aligned}
& { }_{0}^{c} D_{t}^{\alpha} f(t)=J^{n-\alpha} D_{t}^{n} f(t) \\
& =\frac{1}{\Gamma(n-\alpha)} \int_{0}^{t}(t-\tau)^{n-\alpha-1} f^{n}(\tau) d \tau
\end{aligned}
$$

Let

$$
L(t)=f^{n}(t)
$$

Taking the Mellin transform of (23) yields

$$
\begin{aligned}
& \mathcal{M}\left({ }_{0}^{c} D_{t}^{\alpha} f(t) ; \omega\right)=\mathcal{M}\left(J^{n-\alpha} D_{t}^{n} f(t)\right) \\
& =\frac{\Gamma(1-\omega-n+\alpha)}{\Gamma(1-\omega)} L(\omega+n-\alpha)
\end{aligned}
$$

where

$$
\begin{aligned}
L(\omega+n-\alpha)= & \sum_{j=0}^{n-1} \frac{\Gamma(1-\omega-n+\alpha+j)}{\Gamma(1-\omega-n+\alpha)} \\
& {\left[f^{n-j-1}(t) t^{w+n-\alpha-j-1}\right]_{0}^{\infty} } \\
& +\frac{\Gamma(1-\omega+\alpha)}{\Gamma(\omega-\alpha)} F(\omega-\alpha)
\end{aligned}
$$

Simplifying (25) further and for $\alpha \in(0,1)$, yields

$$
\begin{aligned}
\mathcal{M}\left({ }_{0}^{c} D_{t}^{\alpha} f(t) ; \omega\right)= & \frac{\Gamma(\alpha-\omega)}{\Gamma(1-\omega)}\left[f(t) t^{\omega-\alpha}\right]_{0}^{\infty} \\
& +\frac{\Gamma(1-\omega-n+\alpha)}{\Gamma(1-\omega)} F(\omega-\alpha)
\end{aligned}
$$

If the function $f(t)$ and $\mathfrak{R}(\omega)$ are such that all substitutions of the limit $t=0$ and $t=\infty$ in (27) equals zero, then (22) is established. Hence, this completes the proof.

\subsection{Mellin transform of the the Miller-Ross sequential frac- tional derivatives}

The Mellin transform of the Miller-Ross sequential fractional derivatives is given in the following result.

Theorem 7. By means of induction, the Mellin transform of the Miller-Ross sequential fractional derivative is given by

$$
\mathcal{M}\left({ }_{0} D_{t}^{\sigma_{n}} f(t) ; \omega\right)=\frac{\Gamma\left(1-\omega-\sigma_{n}\right)}{\Gamma(1-\omega)} F\left(\omega-\sigma_{n}\right)
$$

Hence, (16) is established. 
Proof: For $n=2$, setting $h(t)={ }_{0} D_{t}^{\beta} f(t)$ and by means of the property of the Mellin transform, one gets

$$
\begin{aligned}
\mathcal{M}\left({ }_{0} D_{t}^{\sigma_{2}} f(t) ; \omega\right)= & {\left[{ }_{0} D_{t}^{\alpha_{2}} h(t) t^{\omega-1}\right]_{0}^{\infty} } \\
& +\frac{\Gamma\left(1-\omega+\alpha_{2}\right)}{\Gamma(1-\omega)} H\left(\omega-\alpha_{2}\right)
\end{aligned}
$$

Solving (29) further and rearranging terms yields

$$
\begin{aligned}
& \mathcal{M}\left({ }_{0} D_{t}^{\sigma_{2}} f(t) ; \omega\right)=\left[{ }_{0} D_{t}^{\sigma_{2}-1} f(t) t^{\omega-1}\right]_{0}^{\infty} \\
& +\frac{\Gamma\left(1-\omega+\alpha_{2}\right)}{\Gamma(1-\omega)}\left[{ }_{0} D_{t}^{\sigma_{1}-1} f(t) t^{\omega-\alpha_{2}-1}\right]_{0}^{\infty} \\
& +\frac{\Gamma\left(1-\omega+\sigma_{2}\right)}{\Gamma(1-\omega)} F\left(\omega-\sigma_{2}\right)
\end{aligned}
$$

In general, by means of induction, the Mellin transform of the Miller-Ross sequential fractional derivative is obtained as

$$
\begin{aligned}
\mathcal{M}\left({ }_{0} D_{t}^{\sigma_{n}} f(t) ; \omega\right)= & \sum_{i=1}^{n} \frac{\Gamma\left(1-\omega+\sigma_{n}-\sigma_{i}\right)}{\Gamma(1-\omega)} \\
& \times\left[{ }_{0} D_{t}^{\sigma_{i}-1} f(t) t^{\omega-\sigma_{n}-\sigma_{i}-1}\right]_{0}^{\infty} \\
& +\frac{\Gamma\left(1-\omega+\sigma_{n}\right)}{\Gamma(1-\omega)} F\left(\omega-\sigma_{n}\right)
\end{aligned}
$$

Since the first term of (31) yields zero, therefore (31) reduces to (28). This completes the proof.

\section{Applications}

This section presents three illustrative examples.

\subsection{Application 1}

Solve the following problem via the Mellin transform

$$
t^{2} f^{2}(t)+t^{3} f^{3}(t)=\delta(t-a)
$$

By applying the Mellin transform to both sides of (32), one obtains

$$
\mathcal{M}\left(\left(t^{2} f^{2}(t)+t^{3} f^{3}(t)\right) ; \omega\right)=\mathcal{M}(\delta(t-a) ; \omega)
$$

Using the property of the Mellin transform of the form

$$
\mathcal{M}\left(t^{\alpha} f^{\alpha}(t) ; \omega\right)=\frac{\Gamma(\omega+\alpha)}{\Gamma(\omega)} F(\omega)
$$

and the fact that

$$
\mathcal{M}(\delta(t-a) ; \omega)=a^{\omega-1}
$$

Equation (34) becomes

$$
\frac{\Gamma(\omega+2)}{\Gamma(\omega)} F(\omega)+\frac{\Gamma(\omega+3)}{\Gamma(\omega)} F(\omega)=a^{\omega-1}
$$

Thus,

$$
F(\omega)=\frac{a^{\omega-1} \Gamma(\omega)}{\Gamma(\omega+2)+\Gamma(\omega+3)}
$$

Applying the inverse Mellin transform to (37) by using complex inversion integral to cover the function $f(t)$ explicitly as the solution of (32)

$$
f(t)=\frac{1}{2 \pi i} \int_{b-i \infty}^{b+i \infty} \frac{\Gamma(\omega)}{\Gamma(\omega+2)+\Gamma(\omega+3)} a^{\omega-1} t^{-\omega} d \omega
$$

\subsection{Application 2}

Solve the following problem via the Mellin transform

$$
t^{\frac{3}{2}} f^{\frac{3}{2}}(t)+t^{\frac{5}{2}} f^{\frac{5}{2}}(t)=(\log t)^{\alpha} \delta(t-a)
$$

Taking the Mellin transform of (39) yields

$$
\mathcal{M}\left(\left(t^{\frac{3}{2}} f^{\frac{3}{2}}(t)+t^{\frac{5}{2}} f^{\frac{5}{2}}(t)\right) ; \omega\right)=\mathcal{M}\left((\log t)^{\alpha} \delta(t-a) ; \omega\right)
$$

Once again, using (34) and the fact that

$$
\mathcal{M}\left((\log t)^{\alpha} \delta(t-a) ; \omega\right)=a^{\omega-1}(\log a)^{\alpha}
$$

(40) becomes

$$
\frac{\Gamma\left(\omega+\frac{3}{2}\right)}{\Gamma(\omega)} F(\omega)+\frac{\Gamma\left(\omega+\frac{5}{2}\right)}{\Gamma(\omega)} F(\omega)=a^{\omega-1}(\log a)^{\alpha}
$$

Rearranging terms in (41), one gets

$$
F(\omega)=\frac{a^{\omega-1}(\log a)^{\alpha} \Gamma(\omega)}{\Gamma\left(\omega+\frac{3}{2}\right)+\Gamma\left(\omega+\frac{5}{2}\right)}
$$

Once again, applying the inverse Mellin transform to (43) by using complex inversion integral to cover the function $f(t)$ explicitly as the solution

$$
\begin{aligned}
f(t)= & \frac{1}{2 \pi i} \int_{b-i \infty}^{b+i \infty} \frac{\Gamma(\omega)}{\Gamma\left(\omega+\frac{3}{2}\right)+\Gamma\left(\omega+\frac{5}{2}\right)} \\
& \times a^{\omega-1}(\log a)^{\alpha} t^{-\omega} d \omega
\end{aligned}
$$

Equation (40) is the required explicit solution of (39).

\subsection{Application 3}

Consider the solution of the Caputo fractional derivative of the form

$$
\sum_{k=0}^{1} t_{0}^{\alpha+k}{ }_{0}^{c} D^{\alpha+k} y(t)=f(t)
$$

subject to the initial conditions

$$
y(0)=y^{\prime}(0)=0, y(\infty)=y^{\prime}(\infty)=0
$$

via the Mellin transform.

Applying the Mellin transform to both sides of (45), yields

$$
\frac{\Gamma(1-\omega)(1-\omega-\alpha)}{\Gamma(1-\omega-\alpha)} Y(\omega)=F(\omega)
$$

Therefore,

$$
\frac{\Gamma(1-\omega-\alpha) F(\omega)}{\Gamma(1-\omega)(1-\omega-\alpha)}=Y(\omega)
$$

Equation (48) can be written in a compact form as

$$
F(\omega) H(1-\omega)=Y(\omega)
$$

where

$$
H(1-\omega)=\frac{\Gamma(1-\omega-\alpha)}{\Gamma(1-\omega)(1-\omega-\alpha)}
$$


In the same manner,

$$
H(\omega)=\frac{\Gamma(\omega-\alpha)}{\Gamma(\omega)(\omega-\alpha)}
$$

Equation (51) can be split as follows

$$
\begin{aligned}
& H(\omega)=A(\omega) B(\omega) \\
& A(\omega)=\frac{\Gamma(\omega-\alpha)}{\Gamma(\omega)}
\end{aligned}
$$

and

$$
B(\omega)=\frac{1}{\omega-\alpha}
$$

respectively. The inverse Mellin transform of (53) and (54) are obtained as

$$
A(t)= \begin{cases}\frac{t^{-\alpha}(1-t)^{\alpha-1}}{\Gamma(\alpha)}, & t \in(0,1) \\ 0, & t>1\end{cases}
$$

and

$$
B(t)= \begin{cases}t^{-\alpha}, & t \in(0,1) \\ 0, & t>1\end{cases}
$$

respectively. The inverse Mellin transform of (51) is obtained as

$$
h(t)=\int_{0}^{\infty} A\left(\frac{t}{\tau}\right) B(\tau) \frac{d \tau}{\tau}
$$

From (55) and (56), it follows that

$$
h(t)=\int_{t}^{1} A\left(\frac{t}{\tau}\right) B(\tau) \frac{d \tau}{\tau}, t \in(0,1)
$$

and that

$$
h(t)=0, t>1
$$

The function $H(\omega)$ has a double pole at $\omega=\alpha$ and ordinary poles at $\omega=\alpha-n-1, n \in \mathbb{Z}^{+}$. By means of the residue theorem of the form

$$
\frac{1}{2 \pi i} \int_{\delta \omega} f(\omega) d \omega=\sum_{j=0}^{k} \operatorname{Res}\left(f, \omega_{j}\right), \omega \in \mathbb{C}
$$

one obtains

$$
\begin{aligned}
h(t)= & \int_{\mathcal{P}_{\tau}} H(\omega) t^{-\omega} d \omega \\
= & \frac{1}{2 \pi i} \int_{b-i \infty}^{b+i \infty} H(\omega) t^{-\omega} d \omega \\
= & \sum_{n=0}\left[\operatorname{Res} H(\omega) t^{-\omega}\right]_{\omega=\alpha} \\
& +\sum_{n=0}^{\infty}\left[\operatorname{Res} H(\omega) t^{-\omega}\right]_{\omega=\alpha-n-1}
\end{aligned}
$$

Simplifying (60) further, yields

$$
h(t)=\frac{t^{-\alpha}}{\Gamma(\alpha)}(\ln t+\Xi(\alpha)-\Phi)
$$

$$
+\sum_{n=0}^{\infty} \frac{(-1)^{n} t^{n-\alpha+1}}{(n+1) \Gamma(n+2) \Gamma(\alpha-n-1)}
$$

where $\Phi$ is the logarithmic derivative of the gamma function and the Euler constant $\Xi=0.577215$. The solution of the Caputo fractional differential equation (45) subject to (46) is obtained

$$
y(t)=\int_{0}^{\infty} f(t \tau) h(\tau) d \tau
$$

Equation (62) is known as the Mellin convolution of the functions $f(t)$ and $h(t)$.

\section{Conclusion}

This paper presents the solutions of fractional order equations in the domain of the Mellin transform. The Mellin transform of the Riemann-Liouville fractional derivative, RiemannLiouville fractional integral, Caputo fractional derivative and the Miller-Ross sequential fractional derivative were obtained. Moreover, three fractional order equations were solved via the Mellin transform. Hence, the solutions obtained were recovered by means of the Mellin transform inversion formula.

\section{References}

[1] A. A. Kilbas, H. M. Srivastava \& J. J. Trujillo, "Theory and applications of fractional differential equations", (2006) Elsevier, Amsterdam, Netherland.

[2] A. A. Elbeleze, A. Kilicman and B. M. Taib, "Homotopy pertubation method for fractional Black-Scholes European option pricing equations using Sumudu transform", Mathematical Problems in Engineering, 2013 (2013) 1.

[3] I. Podlubny, Fractional differential equations. An introduction to fractional derivatives, fractional differential equations, to methods of their solution and some of their applications, 198 (1999), Mathematics in Science and Engineering, Academic press

[4] S. E. Fadugba, "Closed-Form Solution of Generalized Fractional BlackScholes-Like Equation using Fractional Reduced Differential Transform Method and Fractional Laplace Homotopy Perturbation Method", International Journal of Engineering and Future Technology 16 (2019) 13.

[5] S. Kumar, A. Yildirim, Y. Khan, H. Jafari, K. Sayevand \& L. Wei, "Analytical solution of fractional Black-Scholes European option pricing equation by using Laplace transform", Journal of Fractional Calculus and Applications 2 (2012) 1.

[6] Ch. Friedrich, "Relaxation and retardation functions of the Maxwell model with fractional derivatives", Rheologica Acta 30 (1991) 151.

[7] R. L. Bagley \& R. A. Calico, "Fractional order state equations for the control of viscoelastically damped structures", J. Guidance 14 (1991) 304.

[8] K. S. Miller \& B. Ross, An Introduction to the Fractional Calculus and Fractional Differential Equations, (1993), John Wiley and Sons Inc., New York.

[9] K. B. Oldham, C. G. Zoski, "Analogue instrumentation for processing polarographic data", Electroanal. Chem. 157 (1983) 27.

[10] A. Kiliçman, "A note on Mellin transform and distributions", Math. Comput. Appl. 9 (2004) 65.

[11] A. Erdélyi, W. Magnus, F. Oberhettinger \& F. Tricomi, Tables of integral 